DOI: https://doi.org/10.11144/Javeriana.upsy16-3.epcr

\title{
Envejecimiento en parálisis cerebral, un reto en investigación e innovación: revisión sistemática
}

\section{Ageing on Cerebral Palsy, the Challenge in Research and Innovation: Systematic Review}

\author{
María Yolanda GonzÁlez-Alonso \\ Universidad de Burgos, España \\ ORCID: http://orcid.org/0000-0003-0818-1996 \\ Amelia García Moltó \\ Universidad Complutense de Madrid, España \\ María Mercedes Ovejero Bruna \\ SERMES CRO, España
}

a Autora de correspondencia. Correo electrónico: mygonzalez@ubu.es

Para citar este artículo: González-Alonso, M. Y., García Moltó, A., \& Ovejero Bruna, M. M. (2017). Envejecimiento en parálisis cerebral, un reto en investigación e innovación: revisión sistemática. Universitas Psychologica, 16(3), 1-15. https://doi.org/ 10.11144/Javeriana.upsy16-3.epcr

\section{RESUMEN}

Apenas existen iniciativas de investigación en el ámbito del envejecimiento activo y saludable de las personas con parálisis cerebral. Encontrar soluciones a este reto social requiere una red de excelencia en investigación que contribuya a vivir mejor a toda la sociedad. Se recopiló información recogida en 34 artículos sobre parálisis cerebral y envejecimiento, concretamente sobre necesidades percibidas, calidad de vida y repercusión del envejecimiento en las personas con parálisis cerebral. Los resultados de la revisión destacan la importancia de un enfoque preventivo, para lo cual se requiere investigar sobre las nuevas necesidades y los indicadores de calidad de vida en las personas con parálisis cerebral que envejecen. De esta forma, se puede facilitar la atención integral de este colectivo en esta etapa de la vida. Invertir en investigación e innovación es esencial para el futuro, es la manera de impulsar productos y servicios y así crear una sociedad mejor para todos los ciudadanos.

Palabras clave

calidad de vida; necesidades; atención integral; inclusión social; capacidad; discapacidad

\section{ABSTRACT}

There are hardly any research initiatives in the field of active and healthy ageing for people with cerebral palsy. To find solutions to this social challenge needs a network of excellence in research to provide a better life to all of society. Information from 34 articles on cerebral palsy and aging was analyzed, specifically on perceived needs, quality of life and the impact of aging on people with cerebral palsy. The results of this review highlight the importance of a preventive approach, which requires research on new needs and quality of life indicators in people with cerebral palsy when ageing. This way may facilitate the integral care of this group at this stage of life. Investing in research and innovation is essential for the future, it is the way to promote products and services and thus create a better society for all citizens. 
Keywords

self-determination; multidisciplinary care; social inclusion; capacities; disability

\section{Introducción}

Los informes del Consejo de Europa evidencian que en todos los países de la Unión el envejecimiento de las personas con discapacidad, especialmente de aquellas que requieren una ayuda o asistencia intensiva debido a la naturaleza de su condición, presenta nuevos desafíos a las sociedades.

En la II Asamblea Mundial sobre el Envejecimiento en llevada a cabo en Madrid en el 2002, se recogía la importancia de investigar sobre la respuesta que las personas con discapacidad estaban dando ante el envejecimiento, y la conveniencia de buscar la integración de los programas, intentando atender a las distintas formas de discapacidad (Sancho, 2002). La Organización Mundial de la Salud ([OMS], 2011) recomienda reforzar y apoyar a la investigación sobre discapacidad, para aumentar su comprensión y asignar recursos de manera más eficiente.

Algunos autores (Comité Español de Representantes de Personas con Discapacidad [CERMI], 2012; Rodríguez, 2010; Verdugo, Shalock, Arias, Gómez, \& de Urriés, 2013) han denunciado que no se cuenta con formación suficiente sobre discapacidad y tampoco en cuanto al proceso de envejecimiento en las personas que la experimentan. Es por ello que interesa investigar y determinar los factores biológicos, psicológicos y sociales de los que dependen y que influyen en la calidad de vida de estas personas, y es necesario tenerlos en cuenta para la planificación de servicios, recursos y apoyos adecuados en el proceso de envejecimiento (Aguado, Alcedo, \& Fontanil, 2012). Las necesidades sentidas por las personas con discapacidad tienen que incorporarse a las propuestas de mejora, reto para el que se deben planificar soluciones concretas y eficaces (Aguado, Alcedo, Arias, \& Rueda, 2007; Verdugo et al., 2013).
La Comisión Internacional sobre Derechos de las Personas con Discapacidad (Organización de Naciones Unidas [ONU], 2006), desde su aplicación en 2008, propuso un cambio social para asegurar a todas las personas con discapacidad su plena inclusión en la sociedad, eliminando para ello todas las barreras que impidan su participación activa. Uno de los principales logros de la convención es situar a la discapacidad en el plano de los derechos humanos, lo que permite dar mayor visibilidad a este sector de personas. El modelo social de discapacidad, relacionado con la asunción de ciertos valores intrínsecos a los derechos humanos, aspira a potenciar el respeto por la dignidad humana, la igualdad y la libertad personal, propiciando la inclusión social (Barranco \& Bariffi, 2010). Este modelo se constituye sobre la base de determinados principios como vida independiente, no discriminación, accesibilidad universal, normalización del entorno y diálogo civil, entre otros (Real Decreto Legislativo, 1/2013).

Parálisis cerebral: calidad de vida y envejecimiento

La parálisis cerebral es la causa más frecuente de discapacidad motora en la primera etapa de la vida y el principal motivo de discapacidad física grave. Es un trastorno que aparece en los primeros años y persiste toda la vida, su prevalencia en países desarrollados se estima en 2-2.5 casos por cada 1000 recién nacidos vivos, existiendo datos que indican una tendencia a aumentar debido principalmente a los cuidados sanitarios y sociales y al incremento de niños prematuros y gestaciones múltiples (Colver, Fairhurst, \& Pharoah, 2014).

A lo largo de la historia sobre el estudio de la parálisis cerebral, esta se ha definido y clasificado de formas diferentes (RobainaCastellanos, Riesgo-Rodríguez, \& RobainaCastellanos, 2007), lo que ha constituido una limitación a la hora de interpretar los resultados epidemiológicos, puesto que se necesitan, no solo instrumentos fiables a la hora de establecer 
mediciones y comparaciones (Pueyo \& Verdrell, 2002), sino también partir de conceptos comunes (Camacho, Pallás, de la Cruz, Simón, \& Mateos, 2007).

El grupo de trabajo formado por Rosenbaum, Paneth, Levinton, Goldstein y Bax (2007) presenta su definición sobre parálisis cerebral como un grupo de trastornos permanentes del desarrollo del movimiento y de la postura, que causan limitaciones en la actividad y que son atribuidos a alteraciones no progresivas ocurridas en el desarrollo cerebral del feto o del lactante. Además de los trastornos motores existen alteraciones de la sensación, percepción, cognición, comunicación y conducta, por epilepsia. Concluyen que conviene establecer un enfoque individualizado y multidimensional para identificar el estatus funcional de cada persona y especificar el perfil de sus necesidades. Esta definición agrupa distintas situaciones clínicas y grados de limitación en la actividad, por lo que es conveniente clasificar a las personas con parálisis cerebral mediante niveles o grupos. Los componentes principales utilizados para la clasificación son las anomalías motoras; las deficiencias asociadas que pueden producir incluso mayor limitación en las habilidades para la función o participación en las actividades; la distribución anatómica y hallazgos radiológicos encontrados y la causa y el momento en que el daño ocurrió.

De acuerdo a la agrupación de las principales entidades de Atención a la Parálisis Cerebral de toda España (ASPACE), las personas con dicha enfermedad manifiestan una amplia variedad de consecuencias (Tabla 1) en diferentes ámbitos: motor, lenguaje y comunicación, cognitivo, sensorial, afectivo-social y bienestar y salud (Confederación ASPACE, 2010).

\section{TABLA 1}

Manifestaciones de la parálisis cerebral

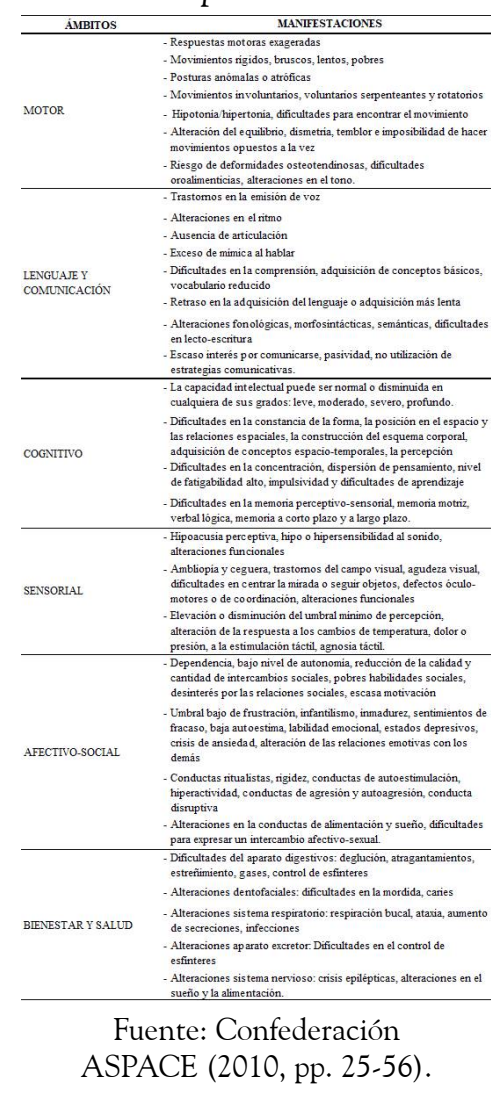

En el colectivo de parálisis cerebral, en la etapa del envejecimiento, las necesidades se acentúan puesto que a la situación que ya conlleva determinadas limitaciones y ajustes, se asocian otros problemas derivados del proceso de envejecimiento. Los efectos del paso del tiempo varían en función de diferentes variables que marcan peculiaridades de su proceso (CERMI, 2012).

Los adultos con parálisis cerebral tienen características y necesidades específicas. En los estudios realizados, se muestran restricciones sociales y también importantes deficiencias musculoesqueléticas y limitaciones progresivas de la marcha (Strauss, Rosenbloom, Shavelle, \& Brooks, 2012).

Schalock y Verdugo (2003) definen calidad de vida en relación con ocho dimensiones de la vida de cada uno: bienestar emocional, relaciones interpersonales, bienestar material, desarrollo personal, bienestar físico, autodeterminación, inclusión social y derechos, que incluyen 
componentes objetivos y subjetivos por valorar en diferentes situaciones de la vida. Desde el modelo de calidad de vida y necesidades percibidas, la satisfacción de las necesidades es un principio primordial para conseguir el bienestar personal de la persona con discapacidad (Verdugo, Navas, Gómez, \& Schalock, 2012).

En materia de discapacidad y envejecimiento apenas hay investigaciones, esto se explica porque hasta hace pocos años, la esperanza de vida de un grupo importante de personas afectadas por deficiencias estaba bastante por debajo de la media de la población general (Blair, Watson, Badawi, \& Stanley, 2001). Las personas con discapacidad no solían llegar a la vejez, pero los avances e innovaciones en las ciencias de la salud, el desarrollo de nuevos apoyos y recursos, la mayor integración social y la mejora de las condiciones de vida han originado el incremento progresivo de personas que llegan a alcanzar edades avanzadas (Brooks et al., 2014). Estas personas experimentan modificaciones respecto a su situación anterior y plantean nuevas necesidades.

El concepto de envejecimiento activo (OMS, 2002) se utiliza como el proceso de optimización de las oportunidades de salud, participación y seguridad con el fin de mejorar la calidad de vida a medida que las personas envejecen. Este planteamiento se basa en el reconocimiento de los derechos humanos de las personas mayores y en los principios de independencia, participación, dignidad, asistencia y realización de los propios deseos. Así, los determinantes pueden ser económicos, sociales, físicos, servicios sociales y de salud, personales (psicológicos y biológicos) y comportamentales (estilos de vida). Para cada persona con parálisis cerebral, la realidad puede ser muy distinta, incluso contar con cierta ventaja puesto que cada persona lleva ya muchos años eliminando y afrontando obstáculos y barreras en su día a día. Así, se plantea la necesidad de dar respuestas individualizadas, desde la interdisciplinariedad en la intervención con profesionales tanto del área de la discapacidad como de la gerontología (Cayo, 2014).
Aceptar estos desafíos relacionados con la parálisis cerebral, con el envejecimiento y con la calidad de vida necesita unos enfoques innovadores en muchos ámbitos de acción y sectores de servicios. Se impone una acción coordinada para satisfacer las necesidades específicas de las personas con discapacidad que envejecen, a fin de permitirles permanecer tanto como sea posible dentro de la sociedad. Esto requiere una evaluación de las necesidades individuales y una planificación prospectiva, tanto como la puesta a disposición de los servicios necesarios. Los problemas y los factores que tienen un efecto desfavorable en la vida diaria de las personas con parálisis cerebral que envejecen y en su participación en las actividades de la sociedad, deberían ser tenidos en consideración en el momento de la concepción de las medidas, estas ya han sido formuladas en el Plan de Acción del Consejo de Europa sobre Discapacidad 2006-2015 y en el Plan de acción de la Estrategia Europea sobre Discapacidad 2010-2020 (Comisión Europea, 2014).

El objetivo del presente estudio es recoger información concreta sobre las personas con parálisis cerebral en proceso de envejecimiento, sus capacidades y necesidades y su calidad de vida. Se ofrece una revisión sistemática que servirá para potenciar la investigación desde la innovación. Mediante este objetivo, se pretende establecer la situación actual que facilite la atención integral de este colectivo en esta etapa de la vida.

\section{Método}

\section{Revisión sistemática}

Se realizó una búsqueda bibliográfica en algunas bases de datos Medline, Scopus, ERIC e ISOC. Para examinar la documentación se aplicó el procedimiento empleado en las revisiones del sistema PRISMA. Los criterios de inclusión utilizados fueron artículos publicados hasta 2016 sobre parálisis cerebral en personas mayores de 18 años, y los criterios de exclusión fueron artículos sobre otra discapacidad en etapa adulta o parálisis 
cerebral en etapa infantil. Para la búsqueda, se utilizaron las palabras claves: cerebral palsy, aging, ageing, elderly, adult, unidas por AND en inglés y parálisis cerebral y envejecimiento en español.

Tras la lectura y revisión de los artículos y aplicando los criterios de inclusión y exclusión, se seleccionó finalmente un total de 34 documentos. El flujo completo de artículos de la búsqueda empleando el sistema PRISMA queda reflejada en la Figura 1 (Moher, Liberati, Altman, $\&$ The PRISMA Group, 2009).

\section{Figura 1.}

Flujo de selección de artículos

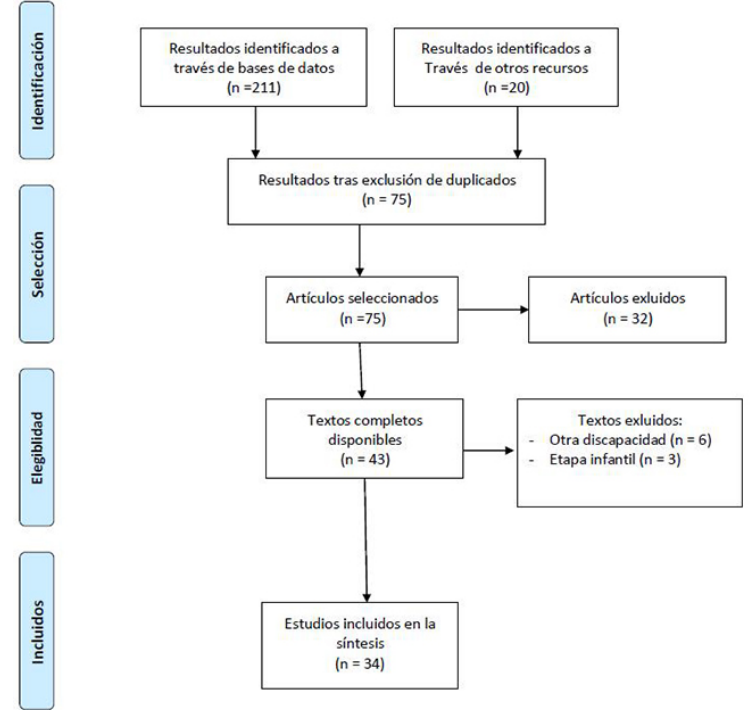

Fuente: elaboración propia.

\section{Resultados}

Los resultados de la revisión sistemática se presentan en la Tabla 2, cada dimensión de calidad de vida se asocia a unos indicadores que reflejan el bienestar o grado de satisfacción personal y las necesidades percibidas, y los artículos en los que aparecen. La mayoría de los artículos encontrados aparecen en revistas de neurología, envejecimiento, discapacidad, rehabilitación y evolutiva.

\section{TABLA 2}

Artículos seleccionados según dimensiones, indicadores de calidad de vida y necesidades percibidas

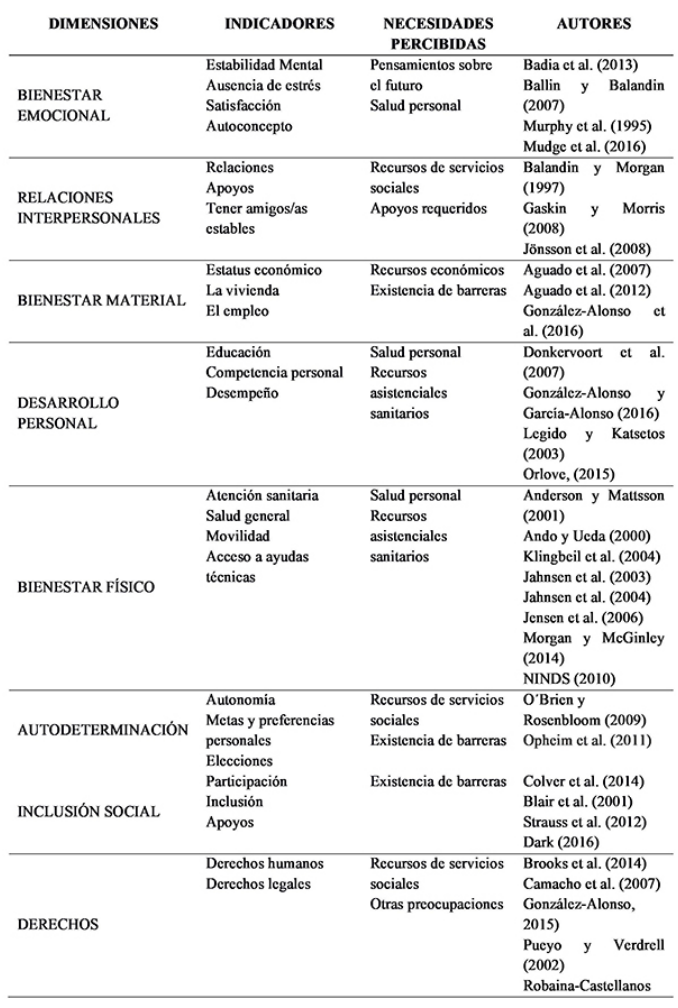

Fuente: elaboración propia a partir de Schalock y Verdugo (2003, pp. 172-174).

\section{Discapacidad y envejecimiento}

El envejecimiento es un cambio importante que requiere nuevas prácticas para seguir ofreciendo apoyos y herramientas (Verdugo \& Schalock, 2013). Se enfatiza la importancia de que las personas con discapacidad actúen de acuerdo a sus intereses personales y demuestren su autodeterminación. La posibilidad de elegir sobre cuestiones personales para hacer frente al presente y planificar el futuro es un elemento importante de la autodeterminación. Incluir a las personas con grave discapacidad, utilizando estrategias con las que se identifiquen o se ayude a identificar estas preferencias, consigue individuos más autodeterminados (Wehmeyer \& Abery, 2013).

Los predictores de un buen envejecimiento son todas aquellas condiciones favorables que 
están presentes en la vida de la persona y auguran una mejor vejez, por lo que prevenir la aparición de enfermedades y discapacidades asociadas ayuda a potenciar factores físicos, psicológicos y de salud. Lograr un envejecimiento satisfactorio y aumentar las posibilidades de envejecer con calidad no es una cuestión de azar, sino más bien un elemento en el que se puede trabajar (Causapié, Balbontín, Porras, \& Mateo, 2011; Fernández-Ballesteros, Robine, Walker, $\&$ Kalache, 2013). Para ello es imprescindible adoptar un enfoque de carácter preventivo y promover en las personas habilidades que potencien comportamientos saludables, tanto en el plano físico, psicológico como social.

Se han realizado investigaciones sobre la aparición de nuevas necesidades (Berzosa, 2013; Navas, Uhlmann, \& Berástegui, 2014; Novell, Nadal, Smilges, Pascual, \& Pujol, 2008) que resaltan la preocupación por el bienestar físico, especialmente por la salud y la asistencia sanitaria, como los indicadores más destacados y determinantes de la calidad de vida en las personas con discapacidad que envejecen. Junto a estos indicadores, el lugar de residencia y sus características, por un lado, y los recursos de apoyo social existentes, el papel de la familia y de los proveedores de servicios, por otro, aparecen como componentes vitales de la calidad de vida en esta población. De hecho, se postula que los efectos del envejecimiento variarán, más que en función de la edad cronológica de los sujetos, en función del estado de salud, de las disfunciones específicas y de la disponibilidad de apoyos y de necesidades cubiertas. Además, la calidad de la atención y el apoyo social son factores contextuales determinantes en este proceso de envejecimiento (Real Decreto Legislativo, 1/2013; OMS, 2011).

\section{Parálisis cerebral y envejecimiento}

Hace décadas era impensable hablar de envejecimiento de las personas con parálisis cerebral. Un diagnóstico precoz y la intervención adecuada durante los años de desarrollo pueden reducir el impacto del paso del tiempo. Con frecuencia, se culpa al diagnóstico global de parálisis cerebral de prácticamente todos los síntomas y problemas que se desarrollan en los adultos y no se hace un esfuerzo por conseguir un diagnóstico específico para síntomas como dolor de piernas, malestar en la espalda, dolor de cabeza (Klingbeil, Baer, \& Wilson, 2004). El objetivo principal debe ser establecer un diagnóstico correcto, ya que el envejecimiento se asocia y se solapa con ciertas condiciones que pueden causar dolor y pérdidas significativas de las funciones (Jahnsen, Villien, Aamodt, \& Stanghelle, 2004; Jahnsen, Villien, Stanghelle, \& Holm, 2003; Jensen, Engel, \& Schwartz, 2006).

La parálisis cerebral espástica dipléjica es el tipo de parálisis cerebral en la que más frecuentemente disminuye la habilidad de caminar con la edad (Anderson \& Mattsson, 2001). Se pierde la marcha antes de los 35 años y se atribuye al dolor en las rodillas, al incremento de la espasticidad, a los problemas de equilibrio y a la ausencia de entrenamiento físico (Morgan \& McGinley, 2014). Desde el punto de vista motriz se observa una disminución en la utilización funcional de sus posibilidades físicas, pérdida de la capacidad de marcha y/o desplazamiento autónomo, dificultad para llevar a cabo una alimentación segura por pérdida de coordinación de la musculatura orofaríngea (Ando \& Ueda, 2000). Las anormalidades del tono muscular desembocan en dolor crónico y deformidades de la cadera y de la espalda.

Entre los principales problemas de salud causados por la lesión de la parálisis cerebral, se encuentra las alteraciones neurológicas que afectan a diferentes estructuras (GonzálezAlonso, 2015; Orlove, 2015); problemas visuales entre los que cabe significar el estrabismo, el nistagmus, la atrofia del nervio óptico, miopía, hemianopsia, ecotoma y cataratas (Legido \& Katsetos, 2003). Los problemas auditivos y las alteraciones en la sensibilidad cutánea y en la propiocepción son frecuentes, y también, las alteraciones perceptuales, principalmente las de estructuración espacial; alteraciones en el lenguaje y comunicación; alteraciones clínicas como la epilepsia y la discapacidad intelectual como consecuencia de la propia 
lesión neurológica o de un trastorno del desarrollo derivado del conjunto de las alteraciones anteriores (Badia, Rodríguez, Orgaz, \& Blanco, 2013; Ballin \& Balandin, 2007). Se asocian con frecuencia complicaciones ortopédicas (contracturas músculo-esqueléticas, luxación de cadera, escoliosis y osteoporosis), problemas digestivos (dificultades para la alimentación, malnutrición, reflujo gastroesofágico, estreñimiento), problemas respiratorios (aspiraciones, neumonías), alteraciones bucodentales, vasculares, etc.

Las consultas de salud por temas generales son infrecuentes. Los problemas médicos comunes de los que normalmente estas personas son tratadas son similares a la población general, como problemas gástricos leves, dolor de cabeza y síndrome premestrual; sin embargo, existe mayor incidencia de infecciones en el tracto urinario e incontinencia, estas infecciones están asociadas a la falta de higiene personal (Murphy, Molnar, \& Lankasky, 1995).

Según Donkervoort, Roebroeck, Wiegerink, Van Der Heijden-Maessen y Stam (2007), los niños con parálisis cerebral que no presentan comorbilidades y reciben una atención médica adecuada tienen una supervivencia similar a la población en general. Algunas secuelas músculo-esqueléticas que van incrementándose con el paso de los años son las deformidades ortopédicas, debilitamiento de los músculos en fuerza y flexibilidad, degeneración general, osteoporosis, fatiga y dolor.

Los estudios sobre adultos concluyen que las personas con parálisis cerebral experimentan cambios negativos en la marcha, digestión, control de la vejiga y de los intestinos, respiración, comunicación y salivación. El Instituto Nacional de Trastornos Neurológicos y Accidentes Cerebrovasculares ([NINDS], 2007) argumenta que los sistemas orgánicos cardiovascular y pulmonar tienen que trabajar más, envejecen prematuramente en la mayoría de las personas con parálisis cerebral, debido al estrés y la tensión.

La depresión, la frustración, la fatiga, la calma son problemas también comúnmente manifestados (Baladin \& Morgan, 1997). La depresión y la ansiedad provocan una reducción del contacto social con impacto sobre la independencia y la interacción social (Gaskin \& Morris, 2008; Jönsson, Ekholm, \& Schult, 2008). La depresión es un trastorno de salud mental frecuente en las personas mayores y también en las personas con parálisis cerebral. Está relacionado no tanto con la gravedad en la afectación de la parálisis cerebral, sino con la forma de enfrentarse a la desilusión y el estrés y si se tiene o no una perspectiva optimista sobre el futuro. Este trastorno puede ser difícil de diagnosticar en algunas personas con parálisis cerebral debido a problemas de comprensión, a dificultades para hablar de sus síntomas y a limitaciones para pedir apoyo emocional. Se ha comprobado que las personas adultas con parálisis cerebral no participan en programas sanitarios preventivos, y que una detección temprana de algunos trastornos de salud podrían ayudar a reducirlos.

Estos cambios físicos, psicológicos y sociales que experimentan las personas con parálisis cerebral en esta etapa de la vida (Tabla 3) requieren nuevas prácticas para un envejecimiento activo que permita el desarrollo de una vida plena.

Hace décadas era impensable hablar de envejecimiento de las personas con parálisis cerebral. Un diagnóstico precoz y la intervención adecuada durante los años de desarrollo pueden reducir el impacto del paso del tiempo. Con frecuencia, se culpa al diagnóstico global de parálisis cerebral de prácticamente todos los síntomas y problemas que se desarrollan en los adultos y no se hace un esfuerzo por conseguir un diagnóstico específico para síntomas como dolor de piernas, malestar en la espalda, dolor de cabeza (Klingbeil, Baer, \& Wilson, 2004). El objetivo principal debe ser establecer un diagnóstico correcto, ya que el envejecimiento se asocia y se solapa con ciertas condiciones que pueden causar dolor y pérdidas significativas de las funciones (Jahnsen, Villien, Aamodt, \& Stanghelle, 2004; Jahnsen, Villien, Stanghelle, \& Holm, 2003; Jensen, Engel, \& Schwartz, 2006).

La parálisis cerebral espástica dipléjica es el tipo de parálisis cerebral en la que más 
frecuentemente disminuye la habilidad de caminar con la edad (Anderson \& Mattsson, 2001). Se pierde la marcha antes de los 35 años y se atribuye al dolor en las rodillas, al incremento de la espasticidad, a los problemas de equilibrio y a la ausencia de entrenamiento físico (Morgan \& McGinley, 2014). Desde el punto de vista motriz se observa una disminución en la utilización funcional de sus posibilidades físicas, pérdida de la capacidad de marcha y/o desplazamiento autónomo, dificultad para llevar a cabo una alimentación segura por pérdida de coordinación de la musculatura orofaríngea (Ando \& Ueda, 2000). Las anormalidades del tono muscular desembocan en dolor crónico y deformidades de la cadera y de la espalda.

Entre los principales problemas de salud causados por la lesión de la parálisis cerebral, se encuentra las alteraciones neurológicas que afectan a diferentes estructuras (GonzálezAlonso, 2015; Orlove, 2015); problemas visuales entre los que cabe significar el estrabismo, el nistagmus, la atrofia del nervio óptico, miopía, hemianopsia, ecotoma y cataratas (Legido \& Katsetos, 2003). Los problemas auditivos y las alteraciones en la sensibilidad cutánea y en la propiocepción son frecuentes, y también, las alteraciones perceptuales, principalmente las de estructuración espacial; alteraciones en el lenguaje y comunicación; alteraciones clínicas como la epilepsia y la discapacidad intelectual como consecuencia de la propia lesión neurológica o de un trastorno del desarrollo derivado del conjunto de las alteraciones anteriores (Badia, Rodríguez, Orgaz, \& Blanco, 2013; Ballin \& Balandin, 2007). Se asocian con frecuencia complicaciones ortopédicas (contracturas músculo-esqueléticas, luxación de cadera, escoliosis y osteoporosis), problemas digestivos (dificultades para la alimentación, malnutrición, reflujo gastroesofágico, estreñimiento), problemas respiratorios (aspiraciones, neumonías), alteraciones bucodentales, vasculares, etc.

Las consultas de salud por temas generales son infrecuentes. Los problemas médicos comunes de los que normalmente estas personas son tratadas son similares a la población general, como problemas gástricos leves, dolor de cabeza y síndrome premestrual; sin embargo, existe mayor incidencia de infecciones en el tracto urinario e incontinencia, estas infecciones están asociadas a la falta de higiene personal (Murphy, Molnar, \& Lankasky, 1995).

Según Donkervoort, Roebroeck, Wiegerink, Van Der Heijden-Maessen y Stam (2007), los niños con parálisis cerebral que no presentan comorbilidades y reciben una atención médica adecuada tienen una supervivencia similar a la población en general. Algunas secuelas músculo-esqueléticas que van incrementándose con el paso de los años son las deformidades ortopédicas, debilitamiento de los músculos en fuerza y flexibilidad, degeneración general, osteoporosis, fatiga y dolor.

Los estudios sobre adultos concluyen que las personas con parálisis cerebral experimentan cambios negativos en la marcha, digestión, control de la vejiga y de los intestinos, respiración, comunicación y salivación. El Instituto Nacional de Trastornos Neurológicos y Accidentes Cerebrovasculares ([NINDS], 2007) argumenta que los sistemas orgánicos cardiovascular y pulmonar tienen que trabajar más, envejecen prematuramente en la mayoría de las personas con parálisis cerebral, debido al estrés y la tensión.

La depresión, la frustración, la fatiga, la calma son problemas también comúnmente manifestados (Baladin \& Morgan, 1997). La depresión y la ansiedad provocan una reducción del contacto social con impacto sobre la independencia y la interacción social (Gaskin \& Morris, 2008; Jönsson, Ekholm, \& Schult, 2008). La depresión es un trastorno de salud mental frecuente en las personas mayores y también en las personas con parálisis cerebral. Está relacionado no tanto con la gravedad en la afectación de la parálisis cerebral, sino con la forma de enfrentarse a la desilusión y el estrés $y$ si se tiene o no una perspectiva optimista sobre el futuro. Este trastorno puede ser difícil de diagnosticar en algunas personas con parálisis cerebral debido a problemas de comprensión, a dificultades para hablar de sus síntomas y a limitaciones para pedir apoyo emocional. Se 
ha comprobado que las personas adultas con parálisis cerebral no participan en programas sanitarios preventivos, y que una detección temprana de algunos trastornos de salud podrían ayudar a reducirlos.

Estos cambios físicos, psicológicos y sociales que experimentan las personas con parálisis cerebral en esta etapa de la vida (Tabla 3) requieren nuevas prácticas para un envejecimiento activo que permita el desarrollo de una vida plena.

\section{TABLA 3}

Sintomas del proceso de envejecimiento en parálisis cerebral

\begin{tabular}{ll}
\hline \multicolumn{1}{c}{ ÁMBITO } & \multicolumn{1}{c}{ SíNTOMAS } \\
\hline SISTEMA CARDIOVASCULAR & - Envejece prematuramente \\
SITEMA RESPIRATORIO & - Utilizansión energia con sensación de fatiga \\
& - Debilidad \\
MOVILIDAD & - Envejece prematuramente \\
& - Disminución de la deambulación \\
SISTEMA DIGESTIVO & - Mayor incidencia de fracturas óseas, \\
SITEMA URINARIO & - Osteoartritis, artritis degenerativa \\
DOLOR & - Reflujo gastroesofägico \\
ESTRUCTURA DE LA FARINGE & - Disfunción vesical \\
ESTRUCTURA DE LA BOCA & - Incontinencia \\
EL OJO & - Mayor sensación de dolor en alguna \\
EL OIDO & - Pificultad para tragar \\
FUNCIONES MENTALES & - Problemas dentales \\
ACTIVIDADES & - Problemas visuales \\
\hline
\end{tabular}

Fuente: elaboración propia.

En algunos centros que atienden a personas mayores con parálisis cerebral se están llevando a cabo nuevas experiencias (Tabla 4) que requieren evaluación y estudio.
TABLA 4

Experiencias de investigación e innovación

\begin{tabular}{|c|c|}
\hline RETOS & $\begin{array}{l}\text { EXPERIENCIAS POSITIVAS } \\
\end{array}$ \\
\hline \multirow{3}{*}{ Accesibilidad } & - Diseño universal en productos de apoyo \\
\hline & - Nuevas tecnologias para la autonomia: instalación de avisadores nocturnos \\
\hline & - Nuevas tecnologias para la inclusión: móvil, cámaras, app, etc. \\
\hline \multirow{5}{*}{ Salud y bienestar } & $\begin{array}{l}\text { - Coordinación sociosanitaria (atención bucodental, médico especialista, } \\
\text { rehabilitador,.... }\end{array}$ \\
\hline & - Proporcionar más cambios posturales \\
\hline & - Acompañamiento en cambios de residencia \\
\hline & - Apoyo en situaciones de duelo \\
\hline & - Alimentación acorde a sus necesidades \\
\hline \multirow{2}{*}{ Participación } & - Grupos de Autogestores \\
\hline & - Redes sociales \\
\hline \multirow{2}{*}{ Comunidad } & - Ocio inclusivo \\
\hline & - Trasporte adaptado \\
\hline \multirow{2}{*}{ Tratamientos } & - Evidencias cientificas \\
\hline & - Aplicación de nuevas tecnologías \\
\hline \multirow{3}{*}{ Familias } & - Información sobre el futuro \\
\hline & - Formación sobre testamento y tutela \\
\hline & - Apoyo emocional \\
\hline \multirow{2}{*}{ Profesionales } & - Formación \\
\hline & - Trabajo en equipo \\
\hline \multirow{3}{*}{ Económicos } & - Convenios \\
\hline & - Subvenciones \\
\hline & - Premios \\
\hline
\end{tabular}

Fuente: elaboración propia.

Sin embargo, todavía falta abordar el proceso de envejecimiento de las personas con parálisis cerebral desde un planteamiento global con modelos teóricos que expliquen dichos procesos. Existen problemas metodológicos sobre la esperanza de vida saludable y en cuanto a las medidas estandarizadas que permitan homologar los resultados de la investigación. Se requieren estudios longitudinales y de cohorte que expliquen cambios generacionales en diferentes condiciones bio-psico-sociales (Fernández-Ballesteros, 2011). Se deben tener en cuenta los múltiples niveles de interacción de las transacciones entre el individuo y el contexto, y realizar estudios desde una perspectiva multidisciplinar que abarque condiciones individuales y también culturales como estereotipos, cuidadores y profesionales que pueden suponer una amenaza para adquirir un envejecimiento saludable (FernándezBallesteros et al., 2013).

\section{Discusión}

En este trabajo se analiza la documentación científica sobre parálisis cerebral y envejecimiento. El objetivo es recoger esta información para potenciar la investigación sobre las personas con parálisis cerebral en proceso de envejecimiento. La investigación sobre el 
envejecimiento ha ido creciendo en los últimos años, según han ido aumentando las cifras de población mayor. En concreto, sobre parálisis cerebral y envejecimiento, a continuación se destacan algunos aspectos obtenidos de los 34 artículos que cumplían los criterios establecidos para la revisión, y que aportan información valiosa que ayudará a abordar esta etapa de la vida para estas personas.

La comunidad científica coincide en la necesidad de mejorar el conocimiento de las características que acontecen en personas que han vivido casi toda su vida con una discapacidad y llegan a alcanzar edades avanzadas, como es el caso de las personas que nos ocupan. Se requiere mejorar el conocimiento de las características psicobiomédicas que acontecen en el proceso de envejecimiento de las personas con discapacidad. También, se resalta la importancia de investigar mejor las necesidades de las personas en su proceso de envejecimiento según la variada tipología de discapacidades y las necesidades de sus familias (González-Alonso, 2015). Igualmente, se deben incrementar, por tanto, las evaluaciones sobre tipos de apoyos y recursos utilizados para cubrir las necesidades en el ámbito de la discapacidad y de las personas mayores, atendiendo a los criterios de flexibilidad y de individualización de cada caso $\left(\mathrm{O}^{\prime}\right.$ Brien \& Rosenbloom, 2009; Opheim, Jahnseb, Olsson, \& Stanghelle, 2011; González-Alonso, SantamaríaVázquez, \& Guijo-Blanco 2016). Se plantea intentar un acercamiento entre profesionales, investigadores, instituciones y entidades del tejido social, para lograr un enriquecimiento mutuo y aportar cada uno su perspectiva tanto a nivel teórico como práctico en materias como la accesibilidad, la coordinación sociosanitaria y las necesidades de prevención, atención y rehabilitación (González-Alonso \& GarcíaAlonso, 2014; Leichsenring, 2005; Real Decreto Legislativo, 1/2013; Rodríguez, Aguado, \& Carpintero, 2003).

Uno de los retos sociales destacados en la Estrategia Europea de Investigación e Innovación (Comisión Europea, 2014) es el envejecimiento. Futurage es un proyecto que desarrolla una hoja de ruta para la investigación en envejecimiento en Europa hasta 2025, a través de un amplio sondeo que destaca dos proyectos: un programa de biotecnología molecular, que establece un plan de trabajo para la investigación europea en los aspectos moleculares hacia un envejecimiento saludable y otro en el campo de las neurociencias dedicado al estudio de enfermedades neurológicas.

El Informe Mundial sobre la Discapacidad (Organización Mundial de la Salud, 2011) recomienda, por una parte, utilizar diferentes ámbitos de investigación, el efecto de los factores ambientales y cómo medirlo, la calidad de vida y bienestar de las personas con discapacidad, y la eficacia y resultados de los servicios y programas para estas personas. Y, por otra parte, plantea formar investigadores especializados y reforzar las aptitudes de investigación en diferentes disciplinas como epidemiología, discapacidad, salud, intervención, educación especial, economía, sociología y política.

Se propone realizar investigaciones sobre la evaluación de los tratamientos que se están utilizando para que profesionales y familias tengan información y así poder elegir la mejor terapia. También, se sugiere investigar los aspectos psicosociales que influyen en la necesidad de apoyo social, autoaceptación y aceptación de los otros y modificaciones para minimizar la fatiga. La Ley General de Derechos de las Personas con Discapacidad y de su inclusión social (2013) resalta las medidas para fomentar la calidad y las medidas de innovación e investigación, aplicadas al desarrollo de entornos, productos, servicios y prestaciones.

La Unión Europea (Comisión Europea, 2014), para abordar los efectos del envejecimiento de la población en el sistema sanitario y la protección social, presenta las siguientes áreas de investigación:

Investigación de la prevención: conservar la salud en el trabajo, efectos de determinados estilos de vida, prevenir caídas, roturas, conservar capacidades, motivar para la gestión de la propia salud y del proceso de envejecimiento y generar un cambio de actitud para adaptarse al entorno social. 
Investigación de enfermedades y terapias: epidemiología, etiología y proceso de envejecimiento, posibilidades de tratamiento de las personas mayores respecto a las enfermedades específicas de la edad.

Investigación sobre la asistencia: nuevas tecnologías, formas óptimas para las prestaciones, cuidados a domicilio, el dolor y los cuidados paliativos.

Investigación de sistemas sanitarios: examinar los sistemas sanitarios y la asistencia de larga duración, aplicación y eficacia de las nuevas tecnologías, y coordinación sociosanitaria.

Otro aspecto para tener en cuenta es la presentación de líneas de investigación según las preferencias de las personas mayores con parálisis cerebral, para diseñar modelos de atención adaptados a sus necesidades; la consecución de ciudades amigables, saludables, accesibles, seguras, sostenibles, ecológicas, inclusivas para las personas mayores con parálisis cerebral siendo las protagonistas de este proceso y las generadoras de bienestar para el resto de la sociedad y la inclusión de iniciativas relacionadas con la importancia de las tecnologías y los productos de apoyo en la construcción de un modelo de sociedad al servicio de las personas (Universidad Complutense, 2011).

Una de las iniciativas enunciadas en la Estrategia Europea (Comisión Europea, 2014) es la unión por la innovación sobre envejecimiento activo y saludable, con el objetivo de mejorar las condiciones de financiación y acceso para la investigación y la innovación, y así afrontar el reto de una población que envejece a fin de garantizar que las ideas innovadoras puedan convertirse en productos y servicios que generen crecimiento y empleo.

La investigación desde una perspectiva psicológica sobre el envejecimiento ha ido creciendo en las últimas décadas, la respuesta de los científicos al análisis del envejecimiento como fenómeno humano y social multifactorial en relación con el bienestar ha tomado mayor protagonismo en los proyectos de investigación de los últimos años.

La esperanza de vida de las personas con parálisis cerebral ha sido poco estudiada, aunque sin duda viven más que en décadas anteriores. Se sabe poco sobre cómo afecta el proceso de envejecimiento a diferentes aspectos como la movilidad, la comunicación, los cambios físicos y emocionales y los cambios relacionados con la edad. Las personas con parálisis cerebral tienen riesgo elevado de adquirir nuevas deficiencias y condiciones de discapacidad en el curso de su vida. Los factores diferenciales o de riesgo son muchos y variados, tanto genéticos como estilos de vida o relaciones sociales, entre otros, que pueden explicar las dificultades y limitaciones añadidas a la discapacidad ya existente. Es necesario conocer los cambios físicos y psicológicos que experimentan las personas con parálisis cerebral en esta etapa del ciclo vital, y sobre todo se deben prevenir sus efectos y aplicar técnicas de intervención para aminorar y responder a las necesidades que acarrean estos cambios.

La perspectiva futura de la investigación sobre parálisis cerebral y envejecimiento plantea estudios sobre el impacto de los cambios a lo largo de la vida en sus aspectos cognitivos y psicosociales, sin perder de vista el aspecto tecnológico para potenciar el desarrollo y aplicaciones en el campo de la estimulación, la comunicación, los servicios domésticos y aplicaciones en los ámbitos de la salud, la asistencia y la inclusión social.

Apoyar esfuerzos internacionales y nacionales encaminados a efectuar investigaciones comparativas sobre el envejecimiento, y asegurar que los datos y evidencias con sensibilidad de género y cuestiones socioculturales estén disponibles para basar en ellos la formulación de las políticas oportunas (ONU, 2012).

Desde el punto de vista metodológico, de acuerdo con el Congreso, Envejecimiento. La investigación en España y Europa (Universidad Complutense, 2011), cuyo objetivo final es el de mejorar la vida de las personas en términos de independencia y autonomía personal, se debe profundizar en la discusión y formalización de conceptos relacionados con parálisis cerebral y envejecimiento desde un punto de vista teórico y echar mano del bagaje científico de múltiples disciplinas, además de 
la psicología, y que los científicos construyan instrumentos que ahonden en una visión multidisciplinar en el campo de los estudios sobre el envejecimiento. Igualmente, se deben combinar métodos cuantitativos y cualitativos de acuerdo a las estrategias de investigaciones e innovación y usar instrumentos habituales y métodos específicos para la recogida de información, fomentar estudios longitudinales y evaluar las medidas utilizadas en los procesos con población mayor. Otras propuestas, teniendo en cuenta aspectos operativos, podrían ser la cooperación y coordinación entre investigadores desde relaciones multidisciplinares y desarrollar una estructura internacional de investigación sobre el proceso de envejecimiento de las personas con parálisis cerebral implicando a los interesados. Se presenta un reto para la investigación sobre envejecimiento que garantice un mundo inclusivo, donde todos podamos vivir una vida de salud, comodidad y dignidad.

\section{Referencias}

Aguado, A. L., Alcedo, M. A., Arias, B., \& Rueda, M. B. (2007). Necesidades de las personas con discapacidad intelectual en proceso de envejecimiento. Bilbao: Diputación Foral de Bizkaia.

Aguado, A. L., Alcedo, M. A., \& Fontanil, Y. (2012). Proceso de envejecimiento en personas con discapacidad intelectual de Castilla y León: calidad de vida y necesidades percibidas. En M. A. Verdugo, R. Canal, M. Badia \& A. L. Aguado (Eds.), Aplicación del paradigma de calidad de vida a la intervención con personas con discapacidad desde una perspectiva integral (pp. 95-121). Salamanca: Publicaciones del INICO.

Anderson, C., \& Mattsson, E. (2001). Adults with cerebral palsy: A survey describing problems, needs and resources, with special emphasis on locomotion. Developmental Medicine and Child Neurology, 43(1), 76-82.

Ando, N., \& Ueda, S. (2000). Functional deterioration in adults with cerebral palsy.
Clinical Rehabilitation, 14, 300-306. http:// dx.doi.org/10.1191/026921500672826716

Badia, M., Rodríguez, P., Orgaz, M. B., \& Blanco, J. M. (2013). Calidad de vida en los pacientes con parálisis cerebral en proceso de envejecimiento. Rehabilitación, 47(4), 194-199. http://dx.doi.org/10.1016/ j.rh.2013.02.002

Balandin, S., \& Morgan, J. (1997). Adults with cerebral palsy: What's happening? Journal of Intellectual and Developmental Disability, 22(2), 109-124. http:// dx.doi.org/10.1080/13668259700033341

Ballin, L., \& Balandin, S. (2007). An exploration of loneliness: Communication and the social networks of older people with cerebral palsy. Journal of Intellectual and Developmental Disability, 32, 315-327. http:// dx.doi.org/10.1080/13668250701689256

Barranco, M. C., \& Bariffi, F. J. (2010). La Convención Internacional sobre los Derechos de las Personas con Discapacidad y su potencial aplicación a las personas mayores (Informe Portal Mayores N. ${ }^{\circ}$ 105). Recuperado del sitio de internet http://www.imsersomayore s.csic.es

Berzosa, G. (2013). Las personas con síndrome de Down y sus familias ante el proceso de envejecimiento. Madrid: Real Patronato sobre Discapacidad, Ministerio de Sanidad, Servicios Sociales e Igualdad.

Blair, E., Watson, L., Badawi, N., \& Stanley, F. J. (2001). Life expectancy among people with cerebral palsy in Western Australia. Developmental Medicine and Child Neurology, 43, 508-515. http://dx.doi.org/10.1111/ j.1469-8749.2001.tb00753.x

Brooks, J. C., Strauss, D. J., Shavelle, R. M., Tran, L. M., Rosenbloom, L., \& Wu, Y. W. (2014). Recent trends in cerebral palsy survival. Part II: individual survival prognosis. Developmental Medicine y Child Neurology, 56, 1065-1071. http:// dx.doi.org/10.1111/dmcn.12519

Camacho, A., Pallás, C. R., de la Cruz, J., Simón, R., \& Mateos, F. (2007). Parálisis cerebral: 
concepto y registros de base poblacional. Revista de Neurología, 45 (8), 503-508.

Causapié, P., Balbontín, A., Porras, M., \& Mateo, A. (2011). Libro blanco sobre envejecimiento activo. Madrid: Ministerio de Sanidad, Política Social e Igualdad.

Cayo, L. (2014). Espacio sociosanitario inclusivo. Madrid. CERMI.

Comité Español de Representantes de Personas con Discapacidad [CERMI] (2012). El envejecimiento de las personas con discapacidad (Documento de posición del CERMI Estatal). Madrid: Autor.

Colver, A., Fairhurst, C., \& Pharoah, P. D. O. (2014). Cerebral palsy. The Lancet, 383(9924), 1240-1249. http://dx.doi.org/10.1016/ S0140-6736(13)61835-8

Comisión Europea. (2014). Comprender las políticas de la Unión Europea: Investigación e innovación. Ginebra. Publicaciones de la Unión Europea.

Confederación ASPACE. (2010). SOMOS: Cuadernos de parálisis cerebral. Madrid: Ministerio de Asuntos Sociales.

Donkervoort, M., Roebroeck, M., Wiegerink, D., Van Der Heijden-Maessen, H., \& Stam, H. (2007). Determinants of functioning of adolescents and young adults with cerebral palsy. Disability Rehabililitation, 29(6), 453-463. http:// dx.doi.org/10.1080/09638280600836018

Comisión Europea. (2014). Estrategia Europea sobre Discapacidad 2010-2020: un compromiso renovado para una Europa sin barreras (Comunicación de la Comisión al Parlamento Europeo, al Consejo, al Comité Económico y Social Europeo y al Comité de las Regiones). Bruselas: Autor.

España, Real Decreto Legislativo 1 de 2013. Por el que se aprueba el Texto Refundido de la Ley General de derechos de las personas con discapacidad y de su inclusión social. Diciembre 3 de 2013. BOE N. ${ }^{\circ} 289$.

Fernández-Ballesteros, R. (marzo, 2011). Envejecimiento saludable. Trabajo presentado en el Congreso sobre envejecimiento. La investigación en España y en Europa. Madrid.

Fernández-Ballesteros, R., Robine, J. M., Walker, A., \& Kalache, A. (2013). Active aging: A global goal [Edición especial]. Current Gerontology and Geriatric Research. A Global Goal. Art ID: 298012. http:// dx.doi.org/10.1155/2013/298012

Gaskin, C. J., \& Morris, T. (2008). Physical activity, health-related quality of life, and psychosocial functioning of adults with cerebral palsy. Journal Physical Act Health, 5(1), 146-157.

González-Alonso, M. Y. (2015). Necesidades sobre salud de los cuidadores de personas con parálisis cerebral en proceso de envejecimiento. En J. J. Gázquez (Coord.), Calidad de vida, cuidadores e intervención para la mejora de la salud en el envejecimiento (pp. 287-294). Almería: Asociación Universitaria de Educación y Psicología.

González-Alonso, M. Y., \& García-Alonso, M. I. (2014). Necesidades sociosanitarias percibidas por las personas con parálisis cerebral en proceso de envejecimiento. Revista INFAD de Psicología. International Journal of Developmental and Educational Psychology, 2(1), 111-120.

González-Alonso, M. Y., Santamaría-Vázquez, M., \& Guijo-Blanco, V. (2016). Perfil de las personas con parálisis cerebral en servicios de vivienda. European Journal of Health Research, 1(3), 83-93. http:// dx.doi.org/10.1989/ejhr.v1i3.8

Instituto Nacional de Trastornos Neurológicos y Accidentes Cerebrovasculares. (2007). Parálisis cerebral: esperanza en la investigación (NIH 07-159s). Bethesda, MD: Autor.

Jahnsen, R., Villien, L., Aamodt, G., \& Stanghelle, J. K., (2004). Musculoskeletal pain in adults with cerebral palsy compared with the general population. Journal of Rehabilitation Medicine, 36(2), 78-84.

Jahnsen, R., Villien, L., Stanghelle, J. K., \& Holm, I. (2003). Fatigue in adults with cerebral palsy in Norway compared with the 
general population. Developmental Medicine and Child Neurology, 45(5), 296-303.

Jensen, M. P., Engel, J. M., \& Schwartz, L. (2006). Coping with cerebral palsy pain: A preliminary longitudinal study. Pain Medicine, 7, 30-37. http://dx.doi.org/10.1111/ j.1526-4637.2006.00086.x

Jönsson, G., Ekholm, J., \& Schult, M. L. (2008). The international classification of functioning, disability and health environmental factors as facilitators or barriers used in describing personal and social networks: A pilot study of adults with cerebral palsy. International Journal of Rehabilitation Research, 31 (2), 119-129. http://dx.doi.org/10.1097/ MRR.0b013e3282fc0f54

Klingbeil, H., Baer, H. R., \& Wilson, P. E. (2004). Aging with a disability. Archives of Physical Medicine and Rehabilitation, 85, 68-73. http://dx.doi.org/10.1016/ j.apmr.2004.03.014

Leichsenring, K. (2005). Proporcionar una asistencia sanitaria y social integrada a las personas mayores. Perspectiva europea. Boletín sobre el envejecimiento, 15. Madrid: Ministerio de Trabajo y Asuntos Sociales. ht tp://riicotec.org/InterPresent2/groups/imse rso/documents/binario/boletinopm15.pdf

Legido, A., \& Katsetos, C. D. (2003). Parálisis cerebral: nuevos conceptos etiopatogénicos. Revista Neurología, 36(2), 157-165.

Moher, D., Liberati, A., Tetzlaff, J., Altman, D. G., \& The PRISMA Group (2009). Preferred Reporting Items for Systematic Reviews and MetaAnalyses: The PRISMA Statement. PLoS Medicine, 6: e1000097. doi:10.1371/journal.pmed1000097.

Morgan, P. P., \& McGinley, J. J. (2014). Gait function and decline in adults with cerebral palsy: A systematic review. Disability y Rehabilitation, 36(1), $\quad 1-9 . \quad$ http:// dx.doi.org/10.3109/09638288.2013.775359

Murphy, K. P., Molnar, G. E., \& Lankasky, K.

(1995). Medical and functional status of adults with cerebral palsy. Developmental Medicine and Child Neurology, 37(12), 1075-1084.

Navas, P., Uhlmann, S., \& Berástegui, A. (2014). Envejecimiento activo y discapacidad intelectual. Madrid: Ministerio de Educación, Cultura y Deporte.

Novell, R., Nadal, M., Smilges, A., Pascual, J., \& Pujol, J. (2008). Informe SENECA: Envejecimiento y discapacidad intelectual en Cataluña 2000-2008 (Informe Ejecutivo). Barcelona: Federación Catalana Pro Personas con Discapacidad Intelectual.

O’Brien, G., \& Rosenbloom, L. (Eds.). (2009). Developmental disability and ageing. Chichester: John Wiley.

Opheim, A., Jahnsen, R., Olsson, E., \& Stanghelle, J. K. (2011). Physical and mental components of health-related quality of life and musculoskeletal pain sites over seven years in adults with spastic cerebral palsy. Journal of Rehabilitation Medicine, 43(5), 382-387. http://dx.doi.org/10.2340/16501977-0787

Organización Mundial de la Salud. (2011). Informe mundial sobre la discapacidad. Ginebra: Autor.

Organización Mundial de la Salud (OMS) (2002). Active Ageing. Ginebra: OMS.

Organización de Naciones Unidas. (2006). Resolución de la Asamblea General Convención internacional amplia e integral para la protección y promoción de los derechos y la dignidad de las personas con discapacidad, A/RES/56/168 (13 de diciembre de 2006). Recuperado de http://www.un.org/spanish/ disabilities/default.asp?id $=1086$

Organización de Naciones Unidas (ONU). (2012). Envejecimiento en el Siglo XXI: una celebración y un desafío. Londres: HelpAge International. https://www.unfpa.org/sites/ default/files/pub-pdf/Ageing\%20Report\%2 OExecutive\%20Summary\%20SPANISH\% 20Final_0.pdf

Orlove, N. M. (2015). Successful aging: Growing older with a developmental disability (Tesis 
doctoral inédita). Universidad de Toledo, Toledo.

Pueyo, B., \& Verdrell, P. (2002). Neuropsicología de la PC. Revista Neurológica, 34(11), 1080-1087.

Robaina-Castellanos, G. R., Riesgo-Rodríguez, S., \& Robaina-Castellanos, M. S. (2007). Definición y clasificación de la parálisis cerebral: iun problema ya resuelto? Revista Neurológica, 45(2), 110-117.

Rodríguez, P., Aguado, A., \& Carpintero, A. (2003). Discapacidad y envejecimiento. Investigación y alternativas de intervención en el proceso de envejecimiento de las personas con discapacidad. Madrid: Fundación ONCE, Escuela Libre Editorial.

Rodríguez, P. (2010). Intervenciones en discapacidad, envejecimiento y dependencia. Hacia la definición de un modelo de calidad de vida basado en derechos y dignidad. Autonomía Personal, 1 , 42-55.

Rosenbaum, P., Paneth, N., Levinton, A., Goldstain, M., \& Bax, M. (2007). A report: The definition and classification of cerebral palsy April 2006. Developmental Medicine and Child Neurology, 49, 8-14. http://dx.doi.org/10.1111/ j.1469-8749.2007.tb12610.x

Sancho, M. T. (2002). Por una sociedad para todas las edades. II Asamblea Mundial sobre el Envejecimiento. Revista Española de Geriatría y Gerontología, 37(Supl. Monográfico 2), 1-122.

Schalock, R. L., \& Verdugo, M. A. (2003). The concept of quality of life in human services: A handbook for human service practitioners. Washington, DC: American Association on Mental Retardation.

Strauss, D. J., Rosenbloom, L., Shavelle, R. M., \& Brooks, J. C. (2012). Improved survival in cerebral palsy in recent decades? Developmental Medicine and Child Neurology, 54(9), 867-868. http://dx.doi.org/10.1111/ j.1469-8749.2012.04349.x

Universidad Complutense. (9 de marzo de 2011). Congreso Envejecimiento: la investigación en España y en Europa [Mensaje en un blog]. Recuperado de http://biblioteca.ucm.es/blogs/InfoMat/3 268.php\#.WQ9E_VU1-qY

Verdugo, M. A., Navas, P., Gómez, L. E., \& Schalock, R. L. (2012). The concept of quality of life and its role in enhancing human rights in the field of intellectual disability. Journal of Intellectual Disability Research, 56(11), 1036-1045. http://dx.doi.org/10.1111/ j.1365-2788.2012.01585.x

Verdugo, M. A., \& Schalock, R. L. (2013). El cambio en las organizaciones de discapacidad. Madrid: Alianza.

Verdugo, M. A., Schalock, R. L., Arias, B., Gómez, L., \& de Urriés, B. (2013). Calidad de vida. En M. A. Verdugo \& R. L. Schalock (Coords.), Discapacidad e inclusión manual para la docencia (pp. 443-461). Salamanca: Amarú Ediciones.

Wehmeyer, M. L., \& Abery, B. H. (2013). Self-determination and choice. Intellectual and Developmental Disabilities, 51(5), 399-411. http:// dx.doi.org/10.1352/1934-9556-51.5.399

\section{Notas}

* Artículo de revisión. 